\title{
Gestión de emprendimiento y su incidencia en la promoción del turismo comunitario
}

\author{
Entrepreneurship management and its incidence of community-based \\ tourism promotion
}

Glenda Alejandra Parra Cerezo. ${ }^{1} \&$ Fredy Leonardo Ibarra-Sandoval. ${ }^{2}$

\begin{abstract}
DOI: https://doi.org/10.33262/concienciadigital.v4i4.1.1928

Community-based tourism is part of a productive strategy for a globalized economy and a research object that provides key elements for study within national and international Pymes programs. This research aims to design a tourism entrepreneurship management model to improve the community-based tourism promotion in the Vinces canton of the Los Ríos province. These processes identify conjunctural variables that feed initiatives to take advantage of the agro-industrial productivity of this geographical area, expanding complementary business possibilities with local and foreign strategic allies. The data obtained through surveys carried out with 200 people, dedicated to entrepreneurships in the cacao sector, reinforce the information on quantitative and qualitative indicators of change and border business connectivity with the quality of tourism products. The interviews conducted with community leaders generate controversy over the efforts made to improve the national and foreign market. The communities demand the fulfillment of firm commitments with the State and with international organizations that integrate the cacao market as a policy of economic reactivation through community-based tourism and the generation of a management model. Entrepreneurs say that it's necessary to steer their ideas towards circular projection concepts, in terms of technical advice, framed in the

\footnotetext{
${ }^{1}$ Pontificia Universidad Católica del Ecuador Sede Ambato. Maestría en Gestión de Empresas Turísticas Tungurahua, Ecuador glenda.a.parra.c@ pucesa.edu.ec, https://orcid.org/0000-0002-3721-4673

${ }^{2}$ Pontificia Universidad Católica del Ecuador Sede Ambato. Maestría en Gestión de Empresas Turísticas Tungurahua, Ecuador fibarra@ pucesa.edu.ec, https://orcid.org/0000-0002-7002-0715
} 
synergy of productive businesses complementary with agricultural activities. In Ecuador, community experiences are enhanced by reducing the discriminatory gap on vulnerable groups in recognition of their collective millenary memory.

Keywords: productive strategy, management model, indicators, entrepreneurships.

\section{Resumen}

El turismo comunitario, se enmarca en una estrategia productiva de economía globalizada y un objeto de investigación que proporciona elementos claves para el estudio dentro de programas de Pymes nacionales e internacionales. Esta investigación tiene como objetivo diseñar un modelo de gestión de emprendimiento turístico para el mejoramiento de la promoción del turismo comunitario en el cantón Vinces de la provincia de los Ríos, estos procesos identifican variables coyunturales que alimentan iniciativas para aprovechar la productividad agroindustrial de esta zona geográfica que amplía posibilidades complementarias de negocios con aliados estratégicos locales y extranjeros. Los datos alcanzados mediante encuetas realizadas a 200 personas, dedicadas a emprendimientos en el sector del cacao, refuerzan la información sobre indicadores cuantitativos y cualitativos de cambio y de conectividad empresarial fronteriza con calidad de productos turísticos. Las entrevistas realizadas a los dirigentes comunales generan controversia por la gestión realizada para la mejora del mercado nacional y extranjero, las comunidades exigen el cumplimiento de compromisos en firme con el Estado y con organizaciones internacionales que integren el mercado del cacao como una política de reactivación económica a través del turismo comunitario y la generación de un modelo de gestión. Los emprendedores manifiestan que es necesario conducir sus ideas hacia conceptos de proyección circular, en materia de asesoramiento técnico, enmarcados en la sinergia de negocios productivos, complementarios con las actividades agrícolas. En el Ecuador se realza las experiencias comunitarias reduciendo la brecha discriminatoria sobre los grupos vulnerables en reconocimiento de su memoria milenaria colectiva

Palabras claves: estrategia productiva, modelo de gestión, indicadores, emprendimientos.

\section{Introducción}

El interés por las características de las personas que crean empresas se remonta al siglo XVIII, las personas emprendedoras juegan un papel importante en el desarrollo económico y social de una región, como señala Campo \& Alvarez (2013). El emprendedor es una persona que asume una serie de riesgos y desafía la incertidumbre con miras a obtener un beneficio económico. Se han propuesto definiciones históricosintéticas, centradas en la estabilidad temporal, teniendo en cuenta el porcentaje de acciones en propiedad (Wu, 2016), o aludiendo a la funcionalidad para definir a la persona emprendedora; pero en términos genéricos podríamos decir que, una persona o 
agrupación emprendedora es aquella que busca explotar una oportunidad económica (McGuirk, 2015).

Antes de nada, convine hacer una apreciación que suele pasar desapercibida cuando se habla de emprendimiento o de personas emprendedoras y sirven para despojar palabras que nos ocupan de connotaciones mercantilistas, así como, para delimitar sus significados. En el contexto que nos ocupa, estas acciones dificultosas están vinculadas a la creación de una empresa independientemente de cuál sea su objetivo. No obstante, tenemos que reconocer, una actividad con estas características puede consistir en escribir un libro, tallar una escultura, llevar a cabo una investigación científica, crear una familia o construir una casa con nuestras propias manos; por tanto, quedaría justificada la idea de no considerar, única y exclusivamente a la persona emprendedora como un ente destinado a crear empresas.

El término: turismo comunitario, data sus orígenes en la declaración de Manila, en este evento, se manifiesta la imperiosa necesidad de reconocer los atributos de identidad en algunos territorios, discriminación ejemplificada en la escasa participación que tenían en los planes de desarrollo turístico. La declaración manifiesta en uno de sus acápites: "en el interior de cada país la oferta turística no constituye un enclave aislado, sino que está vinculada a todos los demás sectores de la vida nacional"' (Morgan et al., 2004), palabras que inician una revolución territorial de los pueblos seculares del mundo, en interrelación y coexistencia participativa con otros elementos de la planificación turística, este impulso arremete: "la política de planificación turística debería desarrollarse a los niveles local, regional o nacional, en el marco de la planificación nacional, tales políticas deberían ser objeto de evaluación periódica tanto cuantitativa como cualitativa" (Morgan, 1999). La exclusión de los pueblos queda sin efecto en la planificación del turismo y enaltece el trabajo local en pleno desarrollo. La Carta del Turismo de 1985, acrecienta el interés por el turismo comunitario, en su declaración en el artículo III, que "los Estados deberían: integrar su política turística en su política global de desarrollo a sus diversos niveles: local, regional, nacional e internacional y ampliar la cooperación turística en un marco bilateral, como en uno multilateral, así como en el marco de la Organización Mundial del Turismo (OMT)". Esta declaración dictamina un escenario de multiniveles de planificación, tal como se menciona en el artículo VII: "se invita a las poblaciones que constituyen las comunidades visitadas en los lugares de tránsito y de estancia a ofrecer a los turistas las mejores condiciones de hospitalidad, cortesía y respeto necesarias para el establecimiento de relaciones humanas y sociales armoniosas" (Morgan, 1999). Documentos que visibilizan los recursos naturales y culturales como oferta del nuevo mercado turístico mundial.

Latinoamérica abre nuevos circuitos de complementariedad turística en lo rural. En los años 90 mediante la Asociación de Ecoturismo Comunitario (ASEC), Ecuador despunta por su diversidad pluricultural y biodiversidad, elementos claves que sirven de modelo para estudiar las experiencias realizadas en las provincias de Bolívar, Manabí, Napo, Pastaza, entre otros, estos escenarios constituyen la piedra angular para nuevos estudios de casos en: Canadá, Australia, Nueva Zelanda, Sudáfrica, Namibia y Gran Bretaña, que 
bifurcan campos aleatorios conceptuales como: turismo indígena, turismo étnico, turismo aborigen, ecoturismo comunitario, turismo comunitario, turismo rural comunitario, entre otros, los investigadores turísticos crean nuevas estrategias en desarrollo local, desarrollo comunitario con proyecciones a los futuros emprendimientos del turismo comunitario (Vásconez, 2018).

Además, se menciona que el Ministerio de Turismo logró impulsar en el 2002, en el literal No. 97, la participación e iniciativa indígena y campesina, con su cultura y tradiciones, que han logrado preservar su identidad y proteger su ecosistema (Monitor, 2017).

Dentro del marco de las tendencias turísticas mundiales el turismo comunitario es uno de los que tienen mayor aceptación en el mercado turístico. En el caso del Ecuador comenzó a configurarse con dinamismo, caracterizado el mismo, porque buena parte de las reservas naturales del país se encuentran en manos de las comunidades indígenas, es así que, el turismo sostenible a través de esta modalidad se preocupa del mantenimiento de la diversidad étnica, cultural y biológica del país, además, su desarrollo se encuentra apoyado por actuales proyectos y leyes normativas para su puesta en marcha (Vargas et al., 2017).

El turismo comunitario, desde un enfoque participativo, tiene preámbulos legales en el concierto del turismo mundial y nacional. La Federación Pluricultural de Turismo Comunitario del Ecuador (FEPTE), comienza su funcionamiento en el 2002, al tenor de la búsqueda del cambio progresivo en la calidad de vida de la población, coloca al ser humano como eje primordial del desarrollo, con equidad social, crecimiento económico, equilibrio ecológico, en convivencia de integración con los elementos que planifican las actividades turísticas, en el concilio de estructuras y lineamientos investigativos en la convivencia, la asociatividad comunitaria; puntos fuertes en las características endógenas y exógenas del desarrollo local.

El desarrollo local, se sustenta en iniciativas turísticas de sostenibilidad rural. La dinámica territorial de las comunidades indígenas atomiza las múltiples posibilidades de formar clústeres de apoyo a iniciativas de emprendimiento que están acordonadas a planificaciones previamente establecidas en diferentes ramales de organización local, regional, nacional e internacional. Intensificar el rescate y creación de actividades recreativas y de descanso es el reto de la nueva página del Ecuador multiétnico y biodiverso (Rivera et al., 2019b).

Las investigaciones sobre la asociatividad de las comunidades fluminenses, marcadas en las iniciativas de nuevos productos derivados del cacao fino de aroma, en el sector, es perpendicular, por el asesoramiento técnico local e internacional que promueven la inversión de tecnologías amigables con el medio ambiente y diversifican la comercialización en mercados, particularmente, europeos. En consecuencia, es importante profundizar en estudios que permitan identificar, analizar y aprender sobre los factores que determinan el comportamiento emprendedor orientado a incursionar en mercados internacionales (Rojas et al., 2017). 
Existe un marco de referencia que, en algunos casos sofoca a las economías locales, la economía mundial; elemento de control sistemático sobre economías emergentes de importaciones y exportaciones, que revitaliza la industria en un orden geométrico cualitativo, esta expansión responde a intereses competitivos de mercado y la transparencia en las regulaciones del sistema bancario mundial, que influye en las políticas del medio ambiente natural, social y otros sectores, contralados por estos organismos unilaterales. La competencia se enraíza y vincula directamente con empresas públicas, privadas, instituciones de educación superior que responden al gregario dominio, dimanante de transnacionales devastadoras. Estos procesos invisibilizan las verdaderas intenciones sobre la liberación de aranceles, desregulación de políticas y la privatización de instituciones caotizadas de imbricaciones políticas concentradas en integrar mercados y no en tejidos sociales.

Las Pymes están menos internacionalizadas que las grandes empresas; además, son cada vez más presionadas por los nuevos mercados internacionales (Lu y Beamish, 2001); de tal manera que en el periodo reciente muchas Pymes tradicionales han decidido acelerar su expansión internacional tanto en economías maduras como en las emergentes (Love \& Roper, 2015). Por ejemplo, en algunas economías emergentes, como en el caso de Latinoamérica, existe una tendencia a incentivar la apertura de los mercados y a promover la internacionalización de las Pymes, sin embargo, la mayoría de los empresarios de esta región no muestran orientación de sus ventas a mercados internacionales (Felzensztein et al., 2015). Es así como, estudios de internacionalización de empresas en países en desarrollo, como las estadísticas del estudio del GEM del año 2015- 2016, indican que existen países con un gran porcentaje de emprendedores (más del 80\%), pero solo con un limitado nivel de orientación internacional, como son los casos de Colombia, Chile, Ecuador y Perú.

En este contexto, diversos estudios sobre el emprendimiento internacional han abordado tres principales temas de investigación: el emprendedor, el entorno externo empresarial y el proceso emprendedor. En referencia al emprendedor, sus características, a nivel individual, han demostrado ser bastante influyentes en las intenciones emprendedoras; en especial, en las Pymes, las cuales, son asociadas, a menudo, a un empresario individual y a su comportamiento (Baker y Nelson, 2005). Adicional a los factores inherentes al emprendedor, estudios recientes también destacan la importancia de la relación entre emprendimiento y crecimiento (Wright y Stigliani, 2013), exportación e innovación (Ganotakis y Love, 2012), capital humano y exportación (Felzensztein et al., 2015).

Estas relaciones en los Pymes de Latinoamérica empiezan a conformar puntos de gestión empresarial que visibilizan problemas en supervivencia de mercados como: aliados estratégicos para financiamiento, talento humano descontextualizado, débil cultura organizativa, esquemas endebles de configuración; en este campo, las dificultades retrasan la conformación de potenciales mercados internacionales, marginando, en algunos, las oportunidades de expansión. Las exigencias en las políticas empresariales de exportaciones realzan la calidad de los productos y los servicios, generando corredores comerciales sostenibles. En los procesos de gestión, se debería establecer las actividades 
específicas en la parte comercial, manufactura o servicios bases en que se apoyan los clientes para establecer claves de financiamiento, las alianzas para el comercio internacional dependen de la naturaleza inquisitiva macroeconómica y la respuesta que tienen las empresas frente a esta presión, desde una microeconomía popular y solidaria.

No obstante, el desarrollo comunitario involucra directamente a agrupaciones o asociaciones jurídicamente establecidas, encaminadas al trabajo sistemático en infraestructura turística, creación y desarrollo de nuevas fuentes de ingresos, en la promoción de la vida social y cultural; la participación de una comunidad diferente de la mencionada es una oportunidad de experimentar rutinas nuevas e intensas (Rivera et al., 2019a).

Estos emprendimientos tienen que enfrentar retos mucho más diferenciadores, en los cuales, se contemplan los problemas como pérdidas humanas, particularmente por el hambre y enfermedades, esto ha comprometido realizar planificaciones que brinden un desahogo de estima y control humano sobre la coraza de impunidad en sectores realmente que necesitan ayuda de transformación y apoyo a iniciativas de gran importancia (García, 2018).

En consecuencia, es importante profundizar en estudios antropológicos, que permitan identificar, analizar y aprender sobre los factores que determinan el comportamiento emprendedor orientado a incursionar en mercados internacionales, abordando, en particular, la predicción de la probabilidad del emprendimiento internacional de los tomadores de decisiones, teniendo en cuenta sus características personales, sus capacidades y recursos, así como los factores cognitivos referidos a la autopercepción y a la percepción del entorno económico y social.

Todo emprendimiento social incluye tres elementos clave: un objetivo social, una innovación transformadora y un modelo de negocios sostenible. El emprendimiento social, suele darse y tener éxito en los sectores de la economía donde el mercado ha fallado y la acción del Estado es inexistente o ineficaz. Un emprendimiento social, cuando resulta exitoso, puede no solo transformar las vidas de los beneficiarios, sino también la forma de abordar un problema en general (Ramírez, 2008). El estudio de la psicología en el comportamiento del ser humano y el mercado meta compromete nuevas oportunidades de desarrollo cognitivo empresarial.

La personalidad encaja características del emprendedor, como la motivación, cuando alcanzan logros inimaginables para el común de la población, las personas emprendedoras tienen la necesidad altiva de establecer metas complejas identificadas de forma progresiva que sustentan su norma de vida en lo social; la inteligencia, cuando asumen retos para otros difíciles de realizar, la innovación y el fracaso, para sustituirlos en optimismo, autosuficiencia, y creación. Estas acciones de los emprendedores, se fundamentan teóricamente en la gestión de actividades de investigaciones realizadas con anterioridad, permite identificar los factores que influyen en la creatividad de innovaciones productivas con fines de lucro, retribuidos en el bosquejo de productos dentro de un accionar de comercialización que delimita los componentes estructurales en composición del diseño 
de un modelo de gestión de emprendimiento turístico para el mejoramiento de la promoción del turismo comunitario.

Estos procesos podrían mejorar si, se cuenta con un grupo de asesores desde los direcciones de planificación, departamentos de turismo de los GAD’s, la Cámara Provincial de Turismo, las Instituciones de Educación Superior y las Asociaciones Comunitarias, podrían ser en áreas específicas, en nuevos procedimientos que enriquezca el perfil académico profesional en prácticas de tiempo real para la planificación integral, inclusiva y participativa de las empresas públicas en los controles y en el cumplimiento de las normativas turísticas.

La empresa conjunta, como un enfoque actitudinal, amplía las posibilidades del desarrollo humano basado en la asociatividad de productos, bienes, servicios y procesos turísticos reflejados en la convivencia de costumbres, tradiciones y saberes ancestrales que, interrelacionados, diversifican las satisfacción de las necesidades de visitantes y viajeros; dilatan la oferta en mecanismos laborales que minimiza el impacto de la migración sobre la herencia cultural de la población y mitigan el accionar de actividades sostenibles en el medio ambiente natural, incorporando nuevas iniciativas de inversionistas nacionales y extranjeros a la estructura y superestructura del sistema turístico ecuatoriano.

Según los autores Campo \& Yagüe (2011), promoción, significa el logro de los objetivos de la empresa mediante la identificación de las necesidades y de los deseos de los consumidores, satisfaciéndolas mejor que los competidores. Los mercados también varían ampliamente, y la determinación de las preferencias de los consumidores podría ser difícil.

Las herramientas más utilizadas por los participantes para promocionar y difundir los servicios y productos de turismo comunitario son las plataformas de internet y las redes sociales. La mayoría declara contar con sitio web para visibilizar su unidad de negocio. Sin embargo, no están seguros de que sea la manera más eficiente de hacerlo ya que desconocen el alcance real de las mismas (Acerenza, 2012, p.39). Según Burnett (1996), se trata de una parte muy importante del Marketing, que se encuentra estrictamente relacionada con la persuasión, siendo la promoción un intermediario y facilitando la relación entre el distribuidor y el cliente, sirviendo de ayuda para ambas partes, satisfaciendo las necesidades de los involucrados.

El cambio de los patrones de consumo, se ven alterados en la carencia de satisfacción de necesidades, esta identificación permite equilibrar un orden dinámico entre la oferta y demanda a través de análisis de los bienes, servicios, productos y procesos del mercado, además, identificar y mejorar los productos existentes es una estrategia de eslabonamiento con el resto de la economía local, diseñada para mejorar la cultura de servicio, de manera eficiente y transparente, satisfacer las necesidades básicas de visitantes y viajeros, mejor que la competencia, es la verdadera meta de complacencia que consideran los servicios complementarios de calidad como un eje en la promoción turística que transforma esta acción, intermediario entre el sistema turístico y el cliente. 
De acuerdo con Osterwalder \& Pigneur (2012): "un modelo de negocios describe las bases sobre las que una empresa crea, proporciona y capta valor". Un modelo de negocios propicia la dirección, a la cual, se dirige la empresa, en conjunto con todos los involucrados para el desarrollo de la misma. Los autores Baker \& Nelson (2005), definen el modelo como una estrategia de construcción colectiva que oriente el desarrollo del municipio de una forma equilibrada y armónica en todos los sectores productivos, proporcionan herramientas para el descubrimiento y la promoción del talento humano y la vocación productivas locales con criterios de sostenibilidad ambiental, hacia el acceso comunitario, el uso de las tecnologías de la información y la comunicación para la generación de ideas conservadoras que permitan la construcción de iniciativas de emprendimiento social.

Según las dos últimas citas, un modelo de negocios es un proceso que describe procedimientos de una empresa para crear, proporcionar y captar valor, de una forma equilibrada y armónica con los sectores productivos en versatilidad con los recursos endógenos y exógenos de las comunidades

Osterwalder \& Pigneur (2012), mencionan que el modelo de negocios responde a tendencias de mercado, que relaciona e integra elementos en común, mientras que Acerenza (2012), resalta la construcción colectiva de un modelo de negocios en valor de su talento humano y la vocación productiva local

El modelo de negocio Canvas, permite medir la deficiencia de las acciones en el emprendimiento considerando su situación financiera para mejorar los productos de oferta y demanda que se encuentran dentro de la infraestructura de gestión sostenida por los nueve componentes que cubren las principales áreas de una empresa, es decir, los clientes, la oferta y viabilidad económica. Los componentes son: a) socios clave, b) actividades clave, c) recursos clave, d) propuesta de valor, e) relación con los clientes, f) canales de distribución, g) segmento de clientes, h) estructura de costos y i) fuentes de ingreso. Este modelo, aplicado por la Corporación para el Desarrollo, CODESPA (2013), basa su metodología en la experiencia regional andina realizada en poblaciones indígenas de Bolivia, Ecuador y Perú, con el objetivo de brindar a las comunidades la oportunidad de generar recursos propios mediante el fortalecimiento de su organización y de la planificación de actividades en beneficio colectivo. El turismo rural comunitario suma el contacto cultural y la participación de los elementos del turismo rural, promueve la relación intercultural entre el anfitrión, y el turista e implica la intervención activa de la comunidad en la definición, planificación y gestión del proceso turístico. Parte de las utilidades generadas se destinan a la atención de las necesidades comunales de forma equitativa.

Según Reinoso (2017), el enfoque principal del turismo comunitario se articula en elementos como: 1) autogestión de las comunidades, 2) elaboración de productos turísticos que revalorizan la cultura local y mantienen el entorno natural, 3) experiencias vivenciales de los turistas en las comunidades indígenas, 4) comercialización directa por parte de las comunidades y, 5) estrategias de generación de ingresos adicionales a la 
agricultura. El objetivo de este modelo es generar resultados dentro de las comunidades indígenas, en las cuales, se logre la competitividad de las mismas, mediante la generación de beneficios a partir de las actividades turísticas, sirviendo como una experiencia piloto en el que, se muestre el potencial que existe en el turismo comunitario, así como también, el de afianzar la autoafirmación de la identidad, la revalorización cultural, el fortalecimiento socio-organizativo y la gestión del territorio.

Según García (2018), señalan, que un modelo de gestión es una herramienta que puede ayudar a la coordinación de los actores del turismo en el territorio y su aplicación requiere tener como base, un profundo y acabado conocimiento de las variables que son estratégicas para su sustentabilidad (una positiva imagen, por ejemplo) y que por esa razón deben ser identificadas, analizadas y seguidas con una frecuencia y técnicas adecuadas.

Para Pérez \& Gonzales (2013), un modelo de gestión recoge un conjunto de proposiciones relativas a las variables a tener en cuenta a la hora de aplicar los conocimientos y su influencia. Es decir, considera todas las variables existentes y las utiliza como base para formar una guía, que indique cómo realizar los pasos especificados dentro de la administración de una organización.

Reinoso (2017), realizó el diseño de un modelo de gestión turístico para fomentar el desarrollo del turismo comunitario en la provincia de Manabí, Ecuador. En su primera fase establece diagnosticar la situación actual del territorio para el desarrollo del turismo comunitario, tiene en cuenta la identificación de problemas y causas, el inventario de recursos turísticos su jerarquización y una planificación estratégica del territorio. En su segunda fase propone un análisis de la gestión turística de la provincia de Manabí, donde se identifica los procesos de planificación y ordenamiento territorial del Gobierno en relación con el turismo, se analiza la oferta y demanda actual. Y la tercera fase la determinación de un plan estratégico de monitoreo, evaluación e identificación de las competencias y funciones de los involucrados.

El tratamiento del turismo comunitario en las tres regiones continentales del Ecuador refleja las diferencias en su accionar, pero existe una falencia en común en la mayoría de las asociaciones de turismo comunitario, la falta de un modelo de gestión que cumpla con herramientas, estrategias, directrices claras, precisas y verificables a corto, mediano y largo plazo en acuerdos comerciales negociables. La oferta turística se desarrolla y comercializa en un período preliminar de operaciones, después, cae en el olvido de rediseño e innovación hacia sus clientes; la fidelización, se aplica a los consumidores (Vera-Rebollo y Baños, 2010).

Según García (2018), mencionan que el modelo de gestión maneja zonas geográficas del turismo comunitario en consideración de las variables estratégicas de planificación turística paralelos al conocimiento de la administración en sus causas y efectos que determinan evaluaciones periódicas sobre sustentabilidad y el cumplimiento de funciones permanentes entre los actores de la planificación territorial 


\section{Metodología}

Se aplicaron encuestas, no paramétricas, a 200 micro emprendedores entre 25-50 años, que habitan en zonas urbanas y rurales del cantón. La metodología de investigación utilizada se fundamenta en lo descriptivo; destaca las herramientas para medir los resultados de la empresa en su autogestión, productos turísticos, y experiencias comunitarias. Explicativo; enfatiza la metodología de elaboración, y estrategias de comercialización análisis-síntesis, recalca la integración de los elementos de la planificación turística y la satisfacción de las necesidades básicas del turista, con un enfoque cuantitativo y cualitativo como métodos de investigación. En la metodología utilizada se adaptaron indicadores establecidos en contextos urbanos y rurales donde se puede ampliar las capacidades creativas y de vanguardia, limitadas por la pandemia mundial.

\section{Resultados}

Estos estudios despiertan el espíritu emprendedor del ecuatoriano, sumergido en crisis económica y afianzan sus ideas innovadoras de mejorar el índice de empleabilidad nacional. El país posee múltiples niveles de planificación en la región de litoral donde la provincia de los Ríos empieza a realizar un proceso en el crecimiento de emprendimientos de la agroindustria cacaotera.

Los encuestados mencionan que necesitan regular sus actividades mediante asesoramiento técnico y conocer las ventajas, desventajas para incursionar en un nuevo negocio productivo complementando las actividades agrícolas del quehacer diario. La utilización y el dominio de las redes sociales sería una solución a corto plazo, las innovaciones se dan por curiosidad en relación a un mercado meta y un producto de permanencia internacional.

Marzo del 2020, es un mes decisivo, registra el primer caso de COVID-19 en el Ecuador, y en Sudamérica, el mercado de comercialización de cacao tiende a la baja y sobreviven los de mayor tradición, en el cantón Vinces de la Provincia de Los Ríos, se encontraron 75 familias, y 13 emprendimientos derivados del cacao, que sostienen la economía local.

A partir de lo expuesto, el cantón Vinces marca precedentes de inclusión por la diversidad de sus productos agrícolas, en particular el cacao. Productos como: el chocolate, vino, turrón, compotas, entre otros. Estos procesos agroindustriales permiten ampliar los derivados de esta fruta y transformarlos en emprendimientos, que se ofertan en mercados nacionales y extranjeros, para combinar bienes, servicios y productos derivados de la memoria colectiva, que impulsan las acciones y operaciones para ampliar las posibilidades del área comercial del turismo comunitario. Se necesita analizar la problemática: la limitada preparación profesional que fragmenta la planificación interinstitucional entre la empresa pública y privada en disminución del asesoramiento técnico hacia los emprendedores comunitarios, 


\section{Discusión}

El enfoque cuantitativo permite realizar una investigación de carácter descriptivo, el impacto de la creación de micro emprendimientos momentáneos y limitados, sugiere un trato especial en la economía comunitaria y familiar, es un fenómeno escasamente estudiado que dirige las necesidades poblacionales a soluciones parches, con excepciones del manejo adecuado de las tecnologías de información y comunicación en el menor tiempo posible, estos emprendimientos posibilitan la generación de economía auxiliar. Como se muestra en la tabla 1.

\section{Tablas 1}

La agroindustria y las redes sociales

\begin{tabular}{ccc}
\hline $\begin{array}{c}\text { Indicadores dominantes } \\
\text { prioritaria }\end{array}$ & $\begin{array}{c}\text { Porcentaje de aceptación } \\
\text { Acciones procedimentales en } \\
\text { beneficio de la creatividad e } \\
\text { innovación }\end{array}$ \\
\hline $\begin{array}{c}\text { Nuevos emprendimientos } \\
\text { agroindustriales }\end{array}$ & $58 \%$ & $\begin{array}{c}\text { Valor agregado como iniciativa } \\
\text { microempresarial, para servicios }\end{array}$ \\
complementarios
\end{tabular}

Esta es una metodología que propicia la participación de todos los elementos de la planificación turística de la provincia de Los Ríos, de preferencia las comunidades emprendedoras del catón Vinces, el diseño del modelo fue propuesto por iniciativa de las autoridades para consolidar el trabajo mancomunado en realidades actuales en plena representación de intereses en común por la reactivación turística, agroeconómica e industrial a pequeña y gran escala, este trabajo esta interrelacionado con el Instituto de investigaciones y desarrollo de la Universidad Técnica de Babahoyo.

\section{Conclusiones}

- Los países emergentes en América Latina pretenden contrarrestar el problema del desempleo mediante el diseño de modelos de gestión en nuevas formas de mercado y negocios dentro de contextos dominantes como el ecuatoriano, anidando en las comunidades la cultura del emprendimiento. En las actividades turísticas gastronómicas y artesanales en la ciudad de Vinces se ensamblan el conjunto de bienes, servicios, productos y procesos turísticos integrados por el Mintur y la Captur de Los Ríos, que ennoblecen la identidad, objetivos y actividades de una empresa comunitaria, esto permite satisfacer las necesidades del turista desde la conceptualidad del análisis de mercado, el manejo financiero, consolidados en la innovación y la competitividad. 
- Existen estudios científicos sobre emprendimientos gastronómicos en diferentes zonas urbanas y rurales del Ecuador, donde sobresalen experiencias lucrativas, que amplían las fuentes de trabajo, para micro emprendedores que necesitan de reconocimiento, asesoramiento y apoyo estatal inclusive para acceder a préstamos no nobiliarios que procuren una rentabilidad a mediano y largo plazo mediante un sistema justo y equitativo del sistema bancario público y privado, dinamizando el territorio nacional.

- La estructura de emprendimiento radica en la complementariedad de negocios como actividades claves, socios, relaciones con los clientes, productos de calidad y amigable con el medio ambiente, con seguridad en la limpieza y manipulación de alimentos, atención con calidad como propuesta de valor agregado, incluida la estructura de segmentos en el mercado en posición de anclaje de servicios complementarios y no tan competitivos entre los mismos socios, la fuente de ingresos está sujeta hacia el autofinanciamiento de recursos incorporados en un nuevo modelo de negocios.

- El aseguramiento de la cultura organizativa empresarial en la población es arcaico se evaden impuestos, aumenta la competencia aleatoria al emprendimiento en pleno irrespeto a los modelos de gestión que sincronizan la planificación, organización, dirección, coordinación, y control del accionar en curso. La articulación de los emprendimientos con la promoción del turismo comunitario radica en el uso de las redes sociales y plataformas virtuales de comercialización como marco de referencia para la economía de desarrollo local.

\section{Referencias bibliográficas}

Acerenza, M. A. (2012). Promoción Turística un enfoque metodológico. México: Trillas.

Baker, T., \& Nelson, R. E. (2005). Creating something from Nothing: Resource Construction through Entrepreneurial Bricolage. Administrative Science Quarterly 50(3), 329-366.

Burnett, J. J. (1996). Promoción: Conceptos y estrategias. SantaFe de Bogota: McGrawHill.

Campo, S. \& Alvarez, M. D. (2013) "Can tourism promotions influence a country's negative image? An experimental study on Israel's image". Current Issues in Tourism 17(3): 201-219

Campo, S., \& Yagüe, M. (2011). Publicidad y promoción en las empresas turísticas. Madrid: Editorial Sintesis.

CODESPA, F. (2013). Metodología para el fortalecimiento de iniciativas de Turismo Rural Comunitario. RUTAS, 7-58

Felzensztein, C., Ciravegna, L., Robson, P., \& Amorós, J. E. (2015). Networks, entrepreneurial orientation, and internationalization scope: evidence from Chilean 
small and medium enterprises. Journal of Small Business Management, 53(sup1), 145-160.

Ganotakis, P., \& Love, J. H. (2012). Export propensity, export intensity and firm performance: The role of the entrepreneurial founding team. Journal of International Business Studies, 43(8), 693-718.

García, C. R., López, E. V., Alfonso, Y. V., \& López, P. V. (2018). La sostenibilidad del turismo comunitario, desde una óptica milenaria. Ciencia Digital, 2(1), 404-419

Love, J. H., \& Roper, S. (2015). SME innovation, exporting and growth: A review of existing evidence. International small business journal, 33(1), 28-48.

Lu, J. W., \& Beamish, P. W. (2001). The internationalization and performance of SMEs. Strategic management journal, 22(6-7), 565-586.

McGuirk, H., Lenihan, H., \& Hart, M. (2015). Measuring the impact of innovative human capital on small firms' propensity to innovate. Research Policy, 44(4), 965-976.

Monitor, G. E. (2017). Global entrepreneurship monitor. Emprendedor Ecuador (Relatório Nacional): Instituto Ecuatoriano de Calidad Productiva.

Morgan, N. A., Kaleka, A., \& Katsikeas, C. S. (2004). Antecedents of export venture performance: A theoretical model and empirical assessment. Journal of marketing, 68(1), 90-108.

Morgan, N.; Pritchard, A. 1999 Tourism promotion and power. Wiley, London

Osterwalder, A. y Pigneur, Y. (2012). Diseñar modelos de negocio y objetos estratégicos similares: la contribución de SI. Revista de la Asociación de sistemas de información, 14 (5), 3.

Pérez, L., \& Gonzales, R. (2013). Propuesta de un modelo de gestión de la reputación on.

Ramírez, A. (2008). ¿Qué es el emprendimiento social? OIT y Schwab Foundation for Social Entrepreneurship. Recuperado el 3 de enero de 2014 de: http://www.comunicacionparaeldesarrollo.org/media_files/download/emprended urismosocialq\&a.pdf

Reinoso, N. G. (06 de 11 de 2017). Cultur. Obtenido de file:///C:/Users/Sistemas/Downloads/Dialnet-

ModeloDeGestionParaFomentarElDesarrolloTuristicoDe-6311570.pdf

Rivera García, C., Arellano Valencia, D., \& Hurtado, S. (2019a). Trabajo comunitario en el recurso turístico natural cascada Pailón San Jacinto, Parroquia Febres Cordero, Cantón Babahoyo. Explorador Digital, 3(3), 62-76 
Rivera García, C., Izurieta Puente, M., \& Cuadro Saucedo, A. (2019b). Aprovechamiento racional de los recursos naturales y culturales para el turismo comunitario, cantón Montalvo-Los Ríos. Ciencia Digital, 3(3), 129-141

Rojas, A., Sánchez, M. J. N., \& Márquez, A. R. (2017). El emprendimiento internacional en países en desarrollo factores determinantes de la predicción del emprendimiento internacional. Barcelona. Universitat Autònoma.

Vargas, M. V. R., Ocaña, Á. F. O., \& Chaviano, E. L. M. (2017). Modelo para la gestión integrada del turismo comunitario en Ecuador, caso de estudio Pastaza. REVESCO. Revista de Estudios Cooperativos, (123), 250-275.

Vásconez, E. A. C. (2018). Turismo comunitario en América Latina, un concepto en construcción. Siembra, 5 (1), 121-131.

Vera-Rebollo, J. F., \& Baños Castiñeira, C. J. (2010). Renovación y reestructuración de los destinos turísticos consolidados del litoral: las prácticas recreativas en la evolución del espacio turístico.

Wright, M., \& Stigliani, I. (2013). Entrepreneurship and growth. International Small Business Journal, 31(1), 3-22.

Wu, J., Wang, C., Hong, J., Piperopoulos, P., \& Zhuo, S. (2016). Internationalization and innovation performance of emerging market enterprises: The role of host-country institutional development. Journal of World Business, 51(2), 251-263.

\section{【 Ciencia}




\section{PARA CITAR EL ARTÍCULO INDEXADO}

Parra Cerezo, G. A., \& Ibarra-Sandoval, F. L. (2021). Gestión de emprendimiento y su incidencia en la promoción del turismo comunitario. ConcienciaDigital, 4(4.1), 96-110. https://doi.org/10.33262/concienciadigital.v4i4.1.1928

\section{LCiencia}

El artículo que se publica es de exclusiva responsabilidad de los autores y no necesariamente reflejan el pensamiento de la Revista Conciencia Digital.

El artículo queda en propiedad de la revista y, por tanto, su publicación parcial y/o total en otro medio tiene que ser autorizado por el director de la Revista Conciencia Digital.

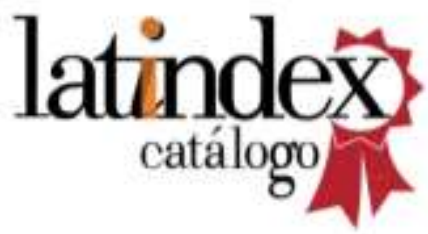

